\title{
Monitoring structural integrity using fibre optic sensors
}

\author{
J Gryzagoridis \\ Mechanical Engineering, University of Cape Town \\ Cape Town, South Africa \\ $+27216503229$ \\ +27216503240 \\ profg@ebe.uct.ac.za \\ F Utou, B Sun \\ Mechanical Engineering Cape Peninsula University of Technology \\ Cape Town, South Africa
}

\begin{abstract}
It is a well known and documented fact that engineering components develop defects and flaws during service which eventually may lead to malfunction or total failure. Typically this would be the result of excessive distortion leading up to the formation and propagation of cracks. The work presented here describes the successful development of a displacement and crack sensing system or sensor, utilizing optical fibres. These were laid equally spaced across a host specimen specially chosen to detect with ease, displacement and the development of a crack. As the crack propagated it opened, which coincided with the particular elongation suffered by each individual optical fibre. As a result of the fibre's elongation, the light passing through it, suffered a loss of intensity which was sensed by specially designed photo detector. An attempt to develop a governing sensor equation is presented. Experiments validate some of the parameters and their relationship however the sensor equation requires further attention. The practical work on this subject accomplished the development of a complete system comprising the sensor, the light emitting and detecting modules. It is a promising method for monitoring structural health.
\end{abstract}

\section{Introduction}

In the past few decades, we have been witnessing drastic changes in materials' technology. Latest developments such as the use of materials with embedded devices have changed the way of thinking in modern design and manufacturing. The incorporated devices, such as sensors and actuators, into a structure form a system which is called a Smart Structure System (SSS) ${ }^{1,2}$

Crack propagation in materials is considered a mechanical fault that needs attention because it may develop as a result of mechanical deformation. In aerospace, aviation, construction, mining and many other industries there are certain structures where, due to dynamic and/or static loading in specific points, mechanical deformation may lead to crack initiation, propagation and subsequent catastrophic failure. Mechanical deformation from fatigue loading has been taken as a major influencing factor in crack creation and propagation. On the other hand, some researchers consider a crack to serve a positive role as a stress relief mechanism (residual stress) ${ }^{3,4}$. 
B.S Jeon et al ${ }^{5}$ have shown that for composite structures, the analysis on crack propagation is complex. Due to the fact that cracks sometimes develop from the inner side (core) of the material, it is difficult to detect or visualize their occurrence, and in most cases to locate their propagation path. Several existing non-destructive testing (NDT) techniques such as magnetic particles detection, acoustic emission, ultrasonic, electronic speckle pattern interferometry (ESPI), shearography, as well as physical visualization, are employed in order to detect crack occurrence and propagation ${ }^{6}$. Recently researchers have been attempting to detect crack orientation using strain as measured with optical fibres ${ }^{7,8}$, The optical fibre has been proven to have reasonably high enough elastic yield when subjected to a tensile force. Optical fibres are also considered to have the mechanical properties, which permit them to withstand deformation under a reasonable extension and as such, when experiencing geometrical changes, it will change the optical radiation characteristics of the light that they may be transmitting through them. Since light is characterized by amplitude (intensity), phase, frequency and polarization, any one of these parameters may be singled out to be monitored as it might undergo changes. The paper reports on the research which aimed to develop a system that could detect and give warning of a crack initiating and propagating within a critical component.

\section{Experimental Procedure}

Bearing in mind the nature of composite materials and our purpose, to detect and monitor crack propagation in composite structures, optical fibres were employed in this study. They formed part of a host specimen comprising the sensor which would be employed to provide the data for the structure's health monitoring system. The single mode optical fibre was considered and selected as most suitable for the embedment in structures. Typically the optical fibre core is made of silica and has a diameter of approximately 125 micrometres and operates in a wave length range of 610 to 770 nanometres. In the sensor, as the optical fibres experienced changes in diameter due to their elongation, the light intensity through the fibres would change and as such recorded by the light detection module. The orientation of the optical fibre embedded into the host structure, (across the intended crack propagation path), provides information for the mechanical deformation for both the optical fibre and host material, provided the fibre is firmly attached to the material. Three optical fibres were placed traversal to the crack's intended direction of travel or propagation at an interval of $2.0 \mathrm{~mm}$ apart. The optical fibres were laid into $1.0 \mathrm{~mm}$ depth grooves, and cemented in place leaving a span or "gauge length" of $4.0 \mathrm{~mm}$ between the bonded ends. The schematic diagram of the test specimen as a failure-warning sensor is shown in figure 1 and was selected as the host structure for the optical fibres, due to the fact that the notch gives a convenient almost assured path for the crack to propagate after initiation. The geometry and dimensions chosen for the specimen were in accordance to the ASTM International Standards E-399-81, E-1820 and BS 7448. The optical fibres extending from both sides of the specimen were terminated with ferrules that connected to their respective light source and photo detector. During testing the crack initiates and propagates along the $\mathrm{x}$ - direction while at the same time the crack widens and the fibres are subjected to extension along the $\mathrm{y}$ - direction. Therefore we observe that the amount of the crack widening or opening has the same value as the extension of the particular 
optical fibre, while its growth or travel along the $\mathrm{x}$ axis was recorded within $2 \mathrm{~mm}$ at 0.1 $\mathrm{mm}$ intervals. The tensile loading of the optical fibre affects its geometry by increasing its length while its diameter decreases.

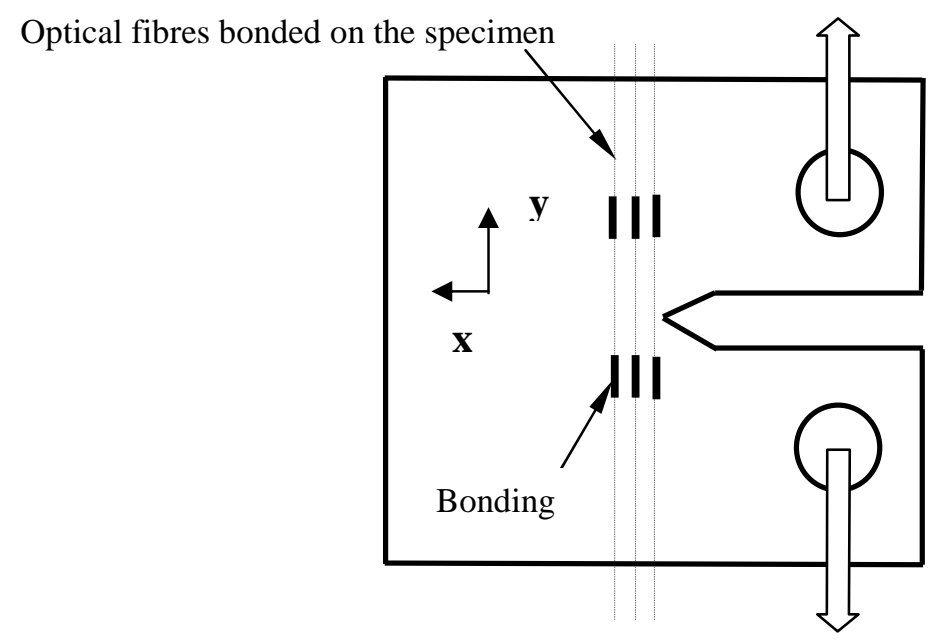

Figure 1. Embedded optical fibres into the test piece

Under the conditions of stretching the optical fibre, while light was being transmitted through it, we note in figure 2 that the light intensity (as voltage output from the photo detector) began to decline until finally it dropped sharply to zero as the optical fibre elongated to $1.7 \mathrm{~mm}$ just prior to fracture. This is in accordance with trends of previously reported work by Takeda Nobuo et al ${ }^{9}$. As the crack tip propagates the region behind it opens wider, therefore the elongation of the fibre that is positioned at a given spot behind the tip of the crack also monitors the crack widening/opening at that position.

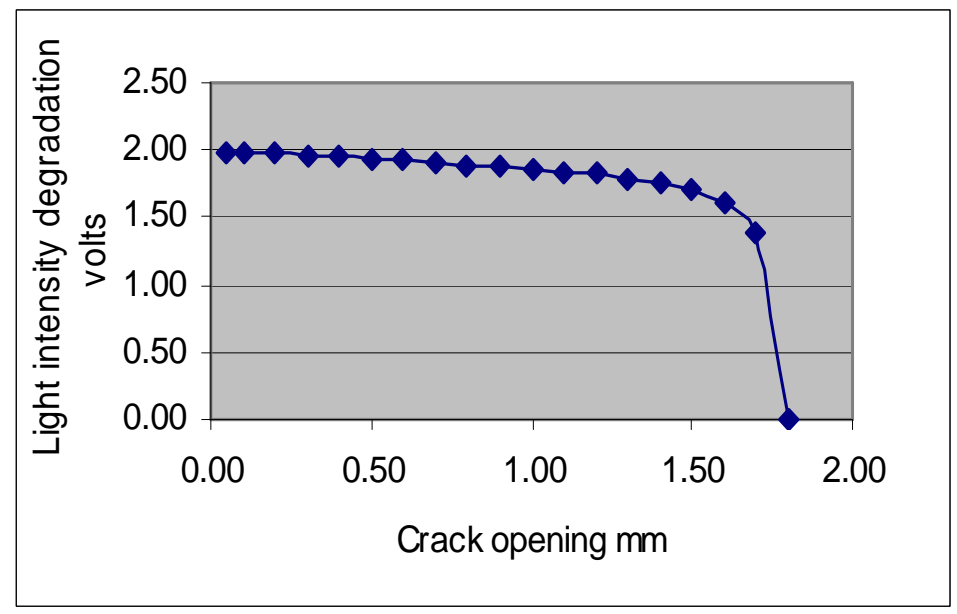

Figure 2. Light intensity degradation through the optical fibre as it elongates 
Similarly the light intensity degradation through the optical fibre as it elongates can be associated with the measured crack length. Elongation of each fibre and therefore crack opening of approximately $1.7 \mathrm{~mm}$ (just before it fails/breaks) corresponds to a total crack length of approximately 26 millimetres long as shown in figure 3 below

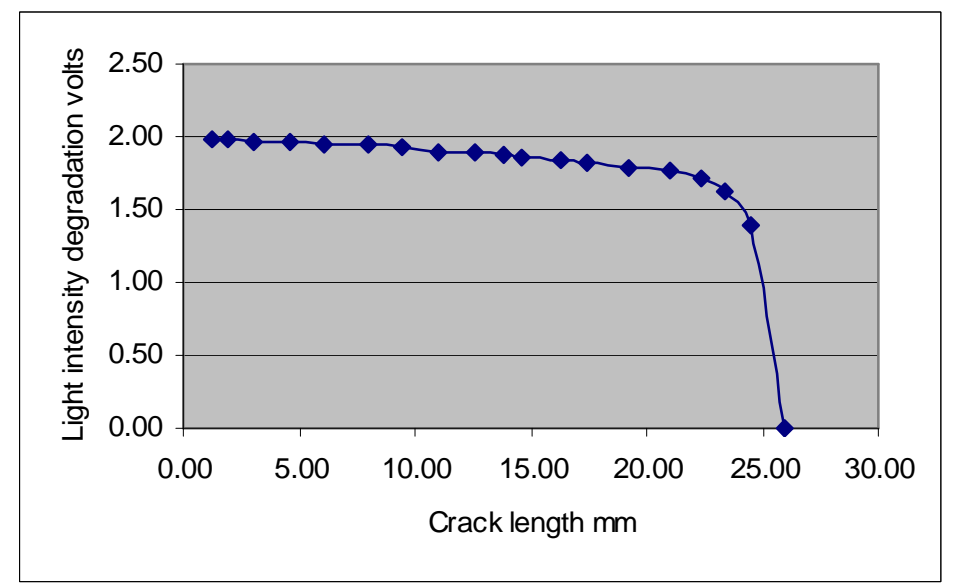

Figure 3. Crack growth as indicated by the light intensity degradation through the optical fibre.

The information obtained from the tests, that is, the degradation of the light intensity as the fibre extended together with the measurements of crack length, afford us the opportunity to depict crack growth vs. crack length as shown in figure 4 below.

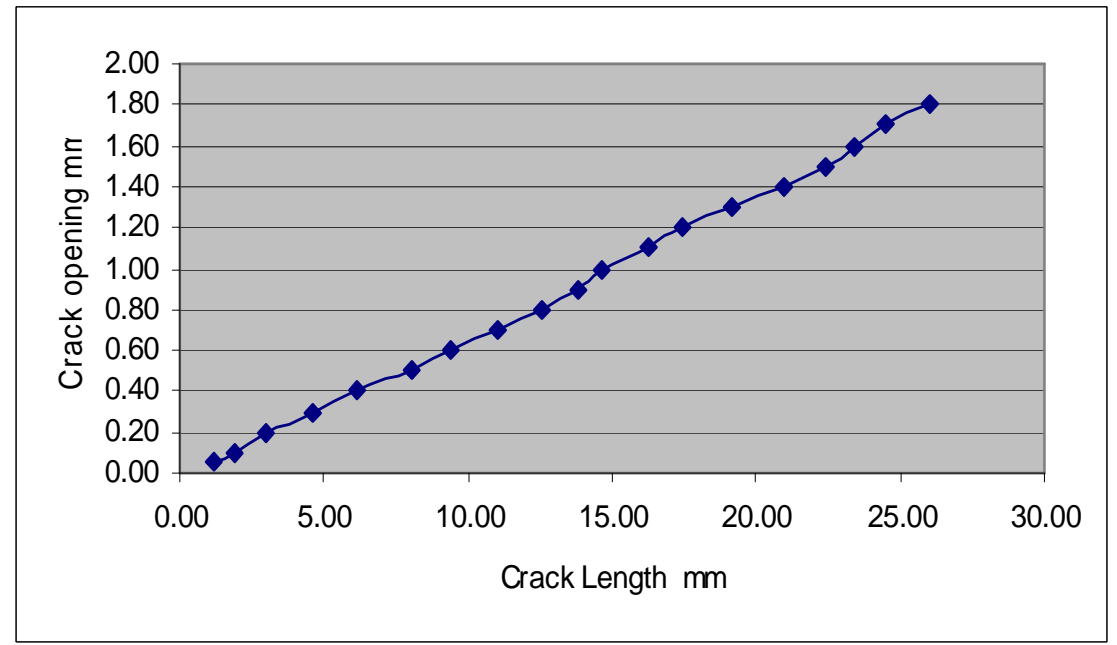

Figure 4. Typical behaviour of crack propagation versus crack opening observed in the fibre host specimen material known as wonder wood 
Typical behaviour of the specimen that hosted the fibre optic cables made from the material known as wonder wood was as expected with the crack initiating at the tip of the notch and propagating along without any appreciable change in direction as shown in figure 5 below.

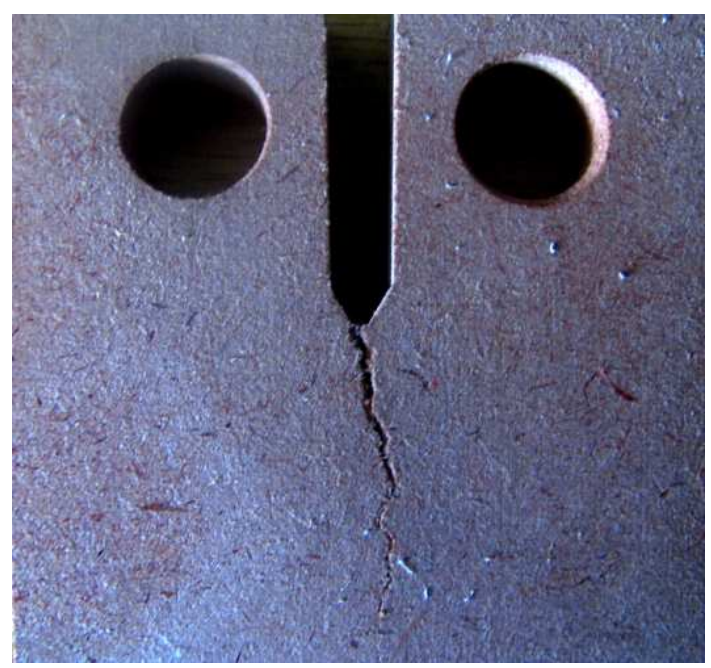

\section{Figure 5. Host specimen made from "wonder wood" depicting typical crack emanating from the tip of the notch.}

\section{Analytical Procedure}

When the optical fibre that is transmitting a light beam is stretched, the light beam encounters the necked section of the fiber, whereby an analogous effect of a reduced aperture occurs. Utou ${ }^{10}$ has shown that, the input - output optical power transmission ratio with respect to the change in optical fiber diameter can be predicted by

$$
\frac{P_{0}}{P_{r}}=\left[1-\left(\frac{\Delta d}{d}\right)^{\alpha}\right]
$$

Where $P_{(r)}$ and $P_{(o)}$ are the optical power through the fiber before and after deformation respectively, $\Delta d$ and $d$ are the reduced and original diameters of the optical fiber and $\alpha$ is the attenuation coefficient per unit length of the optical fibre material. Recalling that the elongation of the optical fibre equals the crack opening of the host specimen (expressed in terms of elastic and plastic behaviour of the host material), at the position where the fibre is fixed, one can obtain a relationship of the optical fibre diameter to the applied load, fibre properties (subscripted with $\mathrm{f}$ ) and physical parameters of the host material ${ }^{10}$ as follows: 


$$
d_{f}=\sqrt{\frac{4 F l}{\pi_{f} E_{f}}\left[\frac{K_{I}^{2}}{2 \sigma E}+\frac{0.4(W-a) v_{p}}{0.4 W+0.6 a}\right]}
$$

The change in optical fiber diameter is obtained by differentiating the above equation with respect to the applied force with all other quantities forming a constant $\mathrm{C}$,

$$
\frac{\Delta d_{f}}{\Delta F_{f}}=\frac{1}{2} \sqrt{C} F^{\frac{-1}{2}}
$$

Combining equations 1 and 3 we obtain an expression which characterizes the optical power output through the fibre that has deformed as a result of the host specimen experiencing a propagating crack.

$$
\frac{P_{0}}{P_{r}}=\left[1-\frac{\Delta F \sqrt{C}}{2 d} \sqrt{\frac{1}{F}}\right]^{\alpha}
$$

Figure 6 below depicts the predicted and experimental values of the optical power ratio through the optical fibre, as it elongates by being fixed across the path of a crack that is propagating and widening in the host specimen.

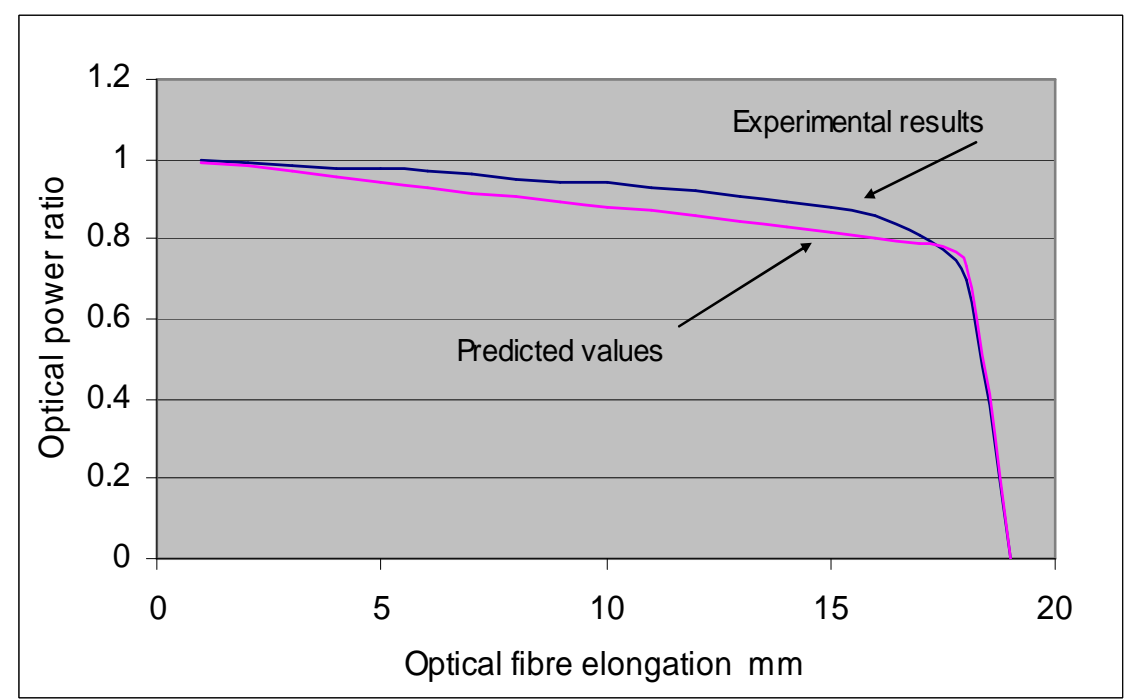

Figure 6. Comparison of experimental and predicted values for the optical power through a fibre subjected to elongation due to an axial force. 


\section{Conclusions and recommendations}

This paper has presented an analysis and experimental work on the development of a sensor to be integrated in a structure. The sensor, based on the principles of fiber optics may be embedded in a critical section of a component monitoring structural health. In particular the sensor by being capable of detecting unwanted excessive distortion, displacement, the initiation and propagation of a crack, presents us with a promising method in warning of impeding catastrophic events. The research on the subject accomplished the development of a complete system including the sensor, the light emitting and detecting modules. While there is a fair degree of confidence in the experimental work that was performed, the theoretical analysis aimed at predicting the optical output power through the optical fibre that has been subjected to dimensional changes, requires further attention toward increasing the accuracy of predicted results. Further work is also envisaged in the process of not only detecting and monitoring crack propagation with optical fibres but also the incorporation of shape memory alloy wires embedded (as part of the host material) to form a smart structure that heals or retards the crack mechanism.

\section{References}

$1 \quad$ M.V Ghandi and B.S Thompson (1992), "Smart Material and Structures" Published by Chapman and Hall-London Chapter 7 pp 2,220

2 W.B Spillman Jr; J.S Sirkis and PT Gardiner, "Smart materials and Structures: What are they?" Journals of Smart Materials and Structures Vol.5 pp. 247

3 Xiao Chun Li, Fritz Prinz and John Seim, "Thermal behaviour of metal embedded fiber Bragg grating sensor", Journals of Smart Materials and Structures (2000) Vol.10 pp. 575

4 E Udd. Fiber Optic Smart Structures Blue Road Research (1994), Chapter 4 pp61-103

5 B.S Jeon et el, "Low velocity impact and delaminating behaviour of composite laminated with fiber optic sensor", Journals of Smart Materials and Structures (1998) vol. 8

6 J. Gryzagoridis, "Safe-live Vs Damage tolerance" 1st National Non-Destructive Testing (NDT) Symposium (Pretoria) (2004).

7 Z.L Yang, G R Liu and K Y Lam, "An inverse procedure for crack detection using integral strain measured by optical fibers". (No $01 \mathrm{pp} 72$ ). Journals of Smart Materials and Structures (2002) vol. 11 pp.72-78

8 Y.C Liang and $\mathrm{C} \mathrm{Hwu,} \mathrm{"On} \mathrm{line} \mathrm{identification} \mathrm{of} \mathrm{holes/cracks} \mathrm{in} \mathrm{composite}$ structures" Journals of Smart Materials and Structures (2001) vol. 10 pp.599-609

9 Takeda Nobuo; Kosaka Tatsuro; Ichiyama Takayuki, Detection of transverse cracks by embedded plastic optical fiber in FRP laminates; Proc. SPIE Vol.3670, p. 248-255, Smart Structures and Materials (1999)

10 Frumence Utou, "Fiber Optic Sensors Ensuring Structural Integrity"; Doctor of Technology thesis, Cape Peninsula University of Technology, Cape Town South Africa. (2005). 